\title{
Coronavirus en el Reino Unido: el costo del excepcionalismo*
}

\section{Coronavirus in the United Kingdom: the cost of exceptionalism}

\section{Paulo Drinot ${ }^{i}$}

'Profesor de historia latinoamericana, Institute of the Americas/University College London. London-UK

orcid.org/0000-0003-3717-1383

paulo.drinot@ucl.ac.uk

Recebido em 8 ago. 2020.

Aprovado em 23 set. 2020.
DRINOT, Paulo. Coronavirus en el Reino Unido: el costo del excepcionalismo. História, Ciências, Saúde - Manguinhos, Rio de Janeiro. Disponível em, Rio de Janeiro, v.28, n.4, out.-dez. 2021, p.1269-1274.

\section{Resumen}

Este artículo ofrece una reflexión sobre las políticas iniciales desarrolladas en el Reino Unido durante la pandemia de SARS-CoV-2.

Palabras clave: coronavirus; Reino Unido; salud pública; pandemia, SARSCoV-2.-2.

Abstract

This article offers a reflection on the policies initially pursued in the United Kingdom during the SARS-CoV-2 pandemic.

Keywords: coronavirus; United Kingdom; public health; pandemic; SARS-CoV-2. 
C suele decir que las pandemias son espejos de una sociedad (Chotiner, 3 mar. 2020). $\checkmark$ Es indudable que la imagen que la pandemia de SARS-CoV-2 refleja del Reino Unido no es nada positiva. Con casi 47 mil muertes oficiales, es el cuarto país más golpeado (después de EEUU, Brasil y México). Según la oficina nacional de estadísticas, la cifra de muertes se avecina a 64 mil. En muertes per capita, solo Bélgica tiene una tasa más alta en Europa. En abril del 2020, el primer ministro, Boris Johnson, del Partido Conservador, fue dado de alta en el hospital St. Thomas donde estuvo internado por varios días despues de contraer el coronavirus. Según informa la prensa, y como él mismo señaló en un mensaje televisivo, estuvo cerca de morir. Se calcula que 540 profesionales de la salud, entre médicos y enfermeros, han muerto. No se puede negar que el confinamiento que se decretó a fines de marzo del 2020 tuvo efecto. Redujo el número de muertos, en la actualidad, a un promedio semanal de menos de cien por día. En abril llegaban a cerca de mil por día (muertes en hospitales, no se contabilizaban las muertes fuera de allì). Pero el costo del manejo de la pandemia, en vidas y en impacto económico, ha sido alto y muchos científicos, entre epidemiólogos y expertos en salud pública, dudan que el país esté adecuadamente preparado para las próximas fases.

¿Pudo ser diferente el manejo inicial de la pandemia? Abundan las comparaciones con Alemania, donde la tasa de mortalidad es mucho menor, donde ya a comienzos de la pandemia había una política de pruebas y de seguimiento de contactos generalizada y donde la capacidad hospitalaria y de cuidados intensivos era mucho mayor (en abril del 2020, había en Alemania casi treinta camas de cuidados intensivos por cien mil habitantes; en el Reino Unido, seis). Las preguntas que surgen son: ¿Cuán bien preparado estaba el Reino Unido para esta pandemia? ¿Cómo ha enfrentado la pandemia desde que se declaró? ¿Y, por último, qué nos dicen tanto el impacto que ha tenido la pandemia como la reacción del gobierno y de la población sobre la sociedad británica? No tendremos respuestas definitivas a las dos primeras preguntas hasta que se haga una investigación oficial pública, que sin duda será en los proximos años. Y, la tercera es materia de reflexión para historiadores y otros científicos sociales del futuro.

Sin embargo, sí es posible hacer algunas observaciones aunque sean algo apresuradas y seguramente superficiales. Mi reflexión busca integrar el contexto de mediano a largo plazo y el contexto de corto plazo. Un buen punto de partida para esta reflexión es la ceremonia de inauguración de los Juegos Olímpicos de Londres de 2012. Estas ceremonias suelen ser usadas por los países anfitriones para presentar al mundo una narrativa sobre sí mismos y mostrar lo que los define. Es por ello interesante que uno de los temas centrales en la ceremonia fue la creación del Servicio Nacional de Salud, o National Health Service (NHS). No es exagerado decir que el NHS es la institución que los británicos valoran por encima de cualquier otra, incluso por encima de la monarquía. Y razón tienen, sobre todo cuando uno mira otros sistemas de salud, en particular el de EEUU o los de América Latina.

Esta valoración del NHS, sin embargo, se entrelaza con otras narrativas nacionales. No debemos olvidar que el NHS se crea a pocos años (1948) de terminada la Segunda Guerra Mundial como consecuencia de un proceso de construcción de instituciones de salud pública que venía gestandose desde fines del siglo XIX (no solo en el Reino Unido) 
y también de las decisiones tomadas por el gobierno de postguerra laborista de Clement Atlee. Se identifica al NHS entonces no solo con un sistema de salud pública definido por ser "exhaustivo, universal y gratuito en el punto de entrega", sino también con un momento de superación y reconstrucción, en el que el país supo reimaginarse tras una tragedia tanto nacional como global. La creación del NHS es, en cierto modo, un momento fundacional de la sociedad británica de la segunda mitad del siglo XX marcado por la Segunda Guerra Mundial.

Sin embargo, la inclusión de este segmento no era solo la celebración del NHS. Era también un desafío para el gobierno de turno. Según el cineasta Danny Boyle, el director de la ceremonia, el gobierno conservador de David Cameron buscó eliminar el segmento de la ceremonia (Osborne, 10 jul. 2016). Elegido en el 2010, el gobierno de Cameron había asumido con fervor la política de austeridad que supuestamente salvaría la economía de las consecuencias de la crisis financiera del 2007-2008. Los recortes de presupuesto tanto al NHS como al sector de "cuidado social" (social care - es decir cuidado a personas de la tercera edad, personas con discapacidades etc.) fueron brutales. Según el British Medical Journal, la política de austeridad de los conservadores añadió 120 mil muertes (Watkins et al., 15 nov. 2017).

Estos recortes se sobrepusieron a una economía y sociedad marcadas por décadas de des-inversión pública, en salud, educación, infrastructura etc., desde los gobiernos de Margaret Thatcher y que los gobiernos laboristas de Tony Blair y Gordon Brown no habían logrado revertir. En regiones del país golpeadas por la des-industrialización, en el norte de Inglaterra, en Gales, en Escocia, en particular, pero en realidad en todo el país, se perfiló una situación sumamente trágica, tal como lo graficó Philip Alston, el rapporteur especial de las Naciones Unidas, cuyo informe sobre la pobreza extrema y los derechos humanos en el Reino Unido concluyó que las políticas de austeridad habían llevado a un "empobrecimiento sistemático" y a un total de 14 millones de personas viviendo en la pobreza (United Nations, 23 abr. 2019).

Es indudable que este contexto ayuda a explicar, en parte, el resultado del referendo del Brexit de 2016. Si bien la decisión de convocar el referendo tenía que ver principalmente con conflictos al interior del Partido Conservador, y no con un rechazo generalizado de la población británica a la Unión Europea (UE), las políticas de austeridad crearon un contexto fértil en el que se potenció aún más un sentimiento anti-europeo y, en particular, anti-inmigrante en algunos sectores de la población británica. Para operadores políticos como Boris Johnson que optaron por la causa del Brexit, el NHS se volvió uno de los campos de batalla: su campaña culpó a los inmigrantes de la UE de poner al NHS bajo presión (aumentando la demanda por sus servicios) y prometió que, al no pagar la cuota de membresía de la UE se podrían destinar 350 millones de libras a la semana al NHS.

Pero, lejos de generar más fondos para el NHS, el resultado del Brexit, y el discurso antiinmigración del gobierno conservador de Theresa May que asumió el poder en el 2016, creó nuevos problemas: se calcula que desde el 2016, 20 mil profesionales de la salud oriundos de países de la UE que trabajaban en el NHS se han ido del Reino Unido (Woodcock, 10 dic. 2019). Si bien estas personas podrían ser remplazadas por migrantes de países que no son miembros de la UE, las políticas migratorias y lo que se ha llamado el "ambiente hostil" 
migratorio (creado por Theresa May cuando era ministra del Interior en 2012) dificultan el reclutamiento de doctores y enfermeras. Según el Nuffield Trust, de aquí al 2030, el Reino Unido tendrá un deficit de 250 mil profesionales de la salud (Rolewicz, Palmer, feb. 2020).

Es indudable que el Reino Unido tiene uno de los mejores sistemas de salud pública en el mundo, pero es un sistema que ha sido erosionado por décadas con políticas de austeridad. Su política de prevención de pandemias ha sufrido por lo mismo: a comienzos de 2020 se calculaba que el material almacenado para ser utilizado en casos de pandemia había perdido el $40 \%$ de su valor (es decir, no ha sido remplazado ni actualizado adecuadamente). Esto, a pesar de que una simulación de pandemia de "gripe de cisne" realizada en el 2016, llamada Exercise Sygnus, concluyó que, de ocurrir una pandemia de esa naturaleza, no habría suficientes ventiladores y material protectivo y que el NHS colapsaría. Es decir, hace cuatro años el gobierno sabía que no estaba preparado para enfrentar una pandemia.

Esto nos lleva al contexto de corto plazo. ¿Cómo explicar la reacción inicial del gobierno de Boris Johnson en la pandemia? A pesar de que los primeros casos en el país se registraron a fines de enero, el gobierno británico no solo demoró en implementar una política de lockdown o confinamiento, como hicieron otros países, sino que por varias semanas parecía que seguiría una política completamente distinta, enfocada no en limitar los contagios a través del confinamiento y seguimiento de casos, sino en permitir que la población adquiriera una imunidad colectiva, la supuesta inmunidad de rebaño. Las recomendaciones del gobierno se limitaron a pedir que la gente se lavara las manos, tosiera en un pañuelo de papel, y se auto-aislara en caso de tener síntomas. A pesar de esta política, la sociedad civil actuó de manera decisiva: las federaciones de futbol y rugby cancelaron partidos, se suspendió la maratón de Londres, y los padres de familia empezaron a retirar a sus hijos de los colegios.

Recién el 18 de marzo se cerraron los colegios y en la última semana de marzo, cuando ya había más de 400 muertos, el gobierno implementó la política de confinamiento. Fueron casi siete semanas desde que se dieron los primeros casos, a fines de enero. El gobierno anunció una serie de medidas económicas importantes tanto para apoyar al NHS (por ejemplo, con la construcción de hospitales temporales para aumentar la cantidad de camas de cuidados intensivos), como a la economía del país; medidas sin precedente, que ampliaban el papel del estado en la economía a niveles no vistos desde la Segunda Guerra Mundial. Sin embargo, las medidas de apoyo económico no cubrieron a toda la población y hubo, en ese momento, problemas de abastecimiento de ventiladores, mascarillas, y otros equipos esenciales. En particular, a pesar de haber prometido aumentar la cantidad de pruebas de coronavirus, por varias semanas, la capacidad continuó siendo baja y limitada en gran parte a pacientes en hospitales. Esto parece haber tenido un impacto nefasto en las casas de retiro (ha habido mejoras en la cantidad de pruebas realizadas, pero el sistema de seguimiento de casos, y en particular el intento de crear una aplicación por teléfono móvil, ha sido un fracaso).

Al comienzo, el gobierno de Johnson insistió que su política divergente se basaba en la "ciencia", a diferencia de otros países, como Italia, donde, según el ministro Grant Shapps, los confinamientos eran medidas "populistas". Este era un mensaje algo contradictorio, ya 
que en los ultimos años el Partido Conservador había asumido un discurso anti-experto en el contexto de su campaña a favor del Brexit. Por otro lado, la política divergente parecía basarse en una idea de la excepcionalidad británica. Lo que se había hecho en otros países no se podía hacer en el Reino Unido, sugirió Boris Johnson, refiriéndose al hecho de que el británico era amante de la libertad. En las primeras semanas, parecía que el gobierno o no entendía o no quería aceptar la seriedad de la pandemia, y mucho menos aceptar que habían lecciones que aprender de la experiencia de otros países (en particular de países asiáticos). No fueron pocos los analistas que reconocieron en esta actitud una cierta soberbia perfumada de nostalgia imperial.

Lejos de asumir responsabilidad tanto por los evidentes errores en las políticas iniciales o por no resolver los problemas de abastecimiento de ventiladores y "equipos de protección personal" (PPE) (en particular, vale insistir, en las casas de retiro de ancianos, donde la pandemia ha tenido un impacto muy grave), el gobierno adoptó una política de comunicación que buscaba socializar la responsabilidad y, por ende, la culpabilidad de las muertes. Su mensaje, repetido ad nauseam, fue "stay at home, protect the NHS, save lives" (quédense en casa, protejan al NHS, salven vidas), haciendo al ciudadano responsable, por sus acciones, de proteger al NHS (la institución tan querida) y salvar las vidas de su conciudadanos. La inhabilidad de asumir la responsabilidad por las deficiencias en la política del gobierno quedó perfectamente ilustrada en la respuesta de la ministra del interior, Priti Patel, a una pregunta de una periodista sobre el desabastecimiento de PPE: "Lo siento si usted piensa que han habido fallas".

A pesar de algunas críticas, incluso de la prensa favorable al gobierno, la mayoría de la población aceptó las medidas de confinamiento y las observó (no así Dominic Cummings, el asesor rasputinesco de Johnson, que se fue de viaje al norte de Inglaterra en pleno lockdown). Al superar y reponerse del coronavirus en abril, Boris Johnson se ganó la simpatía de alguna gente (sigue siendo un primer ministro popular), pero su popularidad, y la de su gobierno, ha ido cayendo a pesar de que la situación, tanto en términos de casos como de muertes, ha mejorado indudablemente (aunque Inglaterra en particular sigue teniendo un nivel de muertes relativamente alto en comparación con otros países de Europa). Mucho dependerá del balance de muertes final y de cómo se manejan las próximas fases de la pandemia, controlando o no rebrotes que parecen ser inevitables, ahora que se ha levantado el confinamiento general. En particular, la vuelta a clases en los colegios y en las universidades en septiembre y octubre presentan un enorme desafío. Es evidente que el confinamiento estricto y general no podía seguir indefinidamente, tanto por el costo que representa para una economía en pausa como por otros tipos de costos (aumentos de violencia doméstica, problemas de salud mental, niños sin acceso a la escolaridad etc.). Del éxito del manejo de las próximas fases de la pandemia dependerá cuan severamente se evaluarán los errores de la política inicial.

\section{NOTA}

* El siguiente texto es una versión más completa, actualizada y sometida a revisión de pares de un post que apareció en el blog de História, Ciências, Saúde - Manguinhos. 


\section{REFERENCIAS}

CHOTINER, Isaac. How pandemics change history. The New Yorker, 3 mar. 2020. Disponible en: https://www.newyorker.com/news/q-and-a/ how-pandemics-change-history. Acceso en: 22 sep. 2020.

OSBORNE, Samuel. Danny Boyle claims Tories tried to axe NHS celebration in London 2012. Olympics opening ceremony. The Independent, 10 jul. 2016. Disponible en: https://www. independent.co.uk/news/uk/politics/dannyboyle-nhs-celebration-tories-london-2012olympics-opening-ceremony-a7129186.html. Acceso en: 22 sep. 2020.

ROLEWICZ, Lucina; PALMER, Billy. The NHS workforce in numbers. NuffieldTrust, 29 feb. 2020. Disponible en: https://www.nuffieldtrust. org.uk/resource/the-nhs-workforce-innumbers \# 8-what-is-the-outlook-for-thefuture. Acceso en: 22 sep. 2020.
UNITED NATIONS. Human Rights Council. Visit to the United Kingdom of Great Britain and Northern Ireland: report of the Special Rapporteur on Extreme Poverty and Human Rights. Geneva: United Nations, 23 abr. 2019. Disponible en: https://digitallibrary.un.org/record/3806308. Acceso en: 22 sep. 2020.

WATKINS, Jonathan et al. Effects of health and social care spending constraints on mortality in England: a time trend analysis, British Medical Journal Open, 15 nov. 2017. Disponible en: https://bmjopen.bmj.com/content/7/11/ e017722. Acceso en: 22 sep. 2020.

WOODCOCK, Andrew. More than 22,000 EU nationals have left NHS since Brexit referendum, figures show. Independent, 10 dic. 2019. Disponible en: https://www.independent. co.uk/news/uk/politics/brexit-eu-citizensnhs-crisis-migration-boris-johnsonhospitalhealth-a9239791.html 7. Acceso en: 22 sep. 2020.

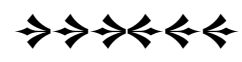

